\title{
Análise espacial como ferramenta de identificação de áreas prioritárias de intervenção para prevenção da sífilis
}

\author{
Spatial analysis as a tool for identification of priority intervention \\ areas for syphilis prevention
}

Roberta de Souza Pereira da Silva Ramos (https://orcid.org/0000-0001-7121-4995) ${ }^{1}$

Vânia Pinheiro Ramos (https://orcid.org/0000-0002-4559-934X) ${ }^{1}$

${ }^{1}$ Departamento de Enfermagem, Universidade Federal de Pernambuco. Av. Prof. Moraes Rego 844900, Cidade Universitária. 50670-420 Recife PE Brasil. roberta_sps@hotmail.com

\begin{abstract}
The spatial analysis of syphilis constitutes a tool capable of contributing to the establishment of guidelines for action in priority geographic areas for preventive intervention. The scope of the article was to describe the scientific evidence that used geoprocessing as a tool to identify risk areas for syphilis. This is an integrative review of the literature, carried out in the Medline/PubMed, Scopus, Web of Science, Lilacs, Ibecs, Cochrane Library Portal, SciELO, Cuiden and Bdenf databases through cross-referencing between the key words "syphilis," "spatial analysis," "geographical information system," "health education" and "geographical mapping." A total of 13 articles were analyzed and in most of them syphilis cases were distributed in a heterogeneous manner, not obeying a unique epidemiological profile in relation to the units of analysis. Discordant ecological and spatial effects between syphilis and HIV and the viability of the integrated screening of syphilis with other diseases was revealed. Efficacy and ability of spatial analysis to target specific educational interventions for each reality were revealed, avoiding investment in geographically non-priority areas for syphilis control.
\end{abstract}

Key words Syphilis, Spatial analysis, Geographic information system and geographical mapping
Resumo A análise espacial da sífilis constitui-se numa ferramenta capaz de contribuir no estabelecimento de diretrizes de atuação nas áreas geográficas prioritárias para intervenções preventivas. $O$ objetivo do artigo foi descrever as evidências produzidas pelos estudos que utilizaram a análise espacial para identificação das áreas prioritárias de intervenção para sífilis. Trata-se de uma revisão integrativa da literatura, realizada nas bases de dados Medline/PubMed, Scopus, Web of science, Lilacs, Ibecs, Portal da Biblioteca Cochrane, SciE$L O$, Cuiden e Bdenf através do cruzamento entre os descritores "sifilis", "análise espacial", "sistema de informação geográfica", "educação em saúde" e "mapeamento geográfico". Foram analisados 13 artigos e na maioria deles os casos de sifilis se apresentaram distribuídos de forma heterogênea, não obedecendo um perfil epidemiológico único em relação as unidades de análise. Foi demonstrado efeito ecológico e espacial discordante entre siffilis e o HIV e a viabilidade no rastreio integrado da sifilis com outras doenças. Foi evidenciado eficácia e capacidade da análise espacial em direcionar intervencões educativas específicas para cada realidade evitando o investimento em áreas geograficamente não prioritárias para o controle da sifilis. Palavras-chave Sifilis, Análise espacial, Sistema de informação geográfica, Mapeamento geográfico 


\section{Introdução}

A sífilis é uma doença infecciosa sistêmica, de evolução crônica, sujeita a surtos de agudização e períodos de latência quando não tratada. É causada pelo Treponema pallidum, patógeno exclusivo do ser humano, de transmissão predominantemente sexual (sífilis adquirida) podendo ocorrer por via materno-fetal (sífilis congênita) ${ }^{1}$.

Apesar da eliminação da sífilis ser uma prioridade global, com propostas de controle articuladas com a Organização Pan-Americana da Saúde (OPAS) e Organização Mundial da Saúde $(\mathrm{OMS})^{2}$ a doença ainda representa um grande desafio para saúde pública em virtude do aumento significativo dos casos diagnosticados. Em 2010, a OMS estimou onze milhões de novos casos de sífilis por ano em todo mundo ${ }^{3}$. Segundo o Ministério da Saúde, em 2013, mais de um milhão de pessoas adquiriram uma infecção sexualmente transmissível, dentre elas a sífilis. No Brasil, apesar do tratamento rápido, no ano de 2010 foram notificados 1.249 casos de sífilis adquirida e em 2015, esses números saltaram para 65.878 notificações, um aumento de mais de $5.000 \%$ em apenas cinco anos configurando uma situação de epidemia ${ }^{4}$.

Falhas no uso do preservativo e resultados pouco expressivos de campanhas de prevenção da doença representam aspectos que contribuem para os números alarmantes da infecção, traduzindo a necessidade de um planejamento de ações de saúde especificamente voltado para as áreas de maior vulnerabilidade para transmissão da doença. Dentro desse contexto, o geoprocessamento representa um conjunto de tecnologias capazes de produzir informações demográficas e contribuir de forma mais eficiente no estabelecimento de diretrizes de atuação e direcionamento de esforços nas áreas geográficas apontadas como zonas de risco para doença ${ }^{5}$.

A proposta de análise espacial da sífilis pode além de mostrar a realidade, facilitar a realização de pesquisas e auxiliar no planejamento e controle da infecção em todas as áreas. Desta forma, obtém-se menor tempo de resposta a epidemia e se reduz custos desnecessários com investimentos em áreas não prioritárias por não representarem zonas de transmissão da infecção. É um recurso que tem sido utilizado por vários autores na área da saúde e seus resultados têm contribuído para a detecção de pontos de transmissão de algumas doenças e para a redefinição da distribuição da rede de assistência de saúde dentro de uma determinada localidade ${ }^{6,7}$.

Considerando a situação epidemiológica da infecção por sífilis no país e no mundo, o objetivo deste estudo foi descrever as evidências produzidas pelos estudos que utilizaram a análise espacial para identificação das áreas prioritárias de intervenção para sífilis.

\section{Método}

Trata-se de uma revisão integrativa da literatura, considerada um método de pesquisa que possibilita a busca, a avaliação crítica e a síntese do estado de conhecimento sobre um determinado assunto, além de apontar lacunas no conhecimento que precisam ser preenchidas com a realização de novos estudos.

Esta revisão foi operacionalizada a partir das seguintes etapas?: 1) estabelecimento da questão norteadora e objetivos da revisão integrativa; 2) definição das bases de dados e das informações a serem extraídas dos artigos selecionados; 3 ) estabelecimento dos critérios de inclusão e exclusão dos artigos (seleção da amostra); 4) análise crítica dos artigos incluídos na revisão; 5) interpretação e discussão dos resultados; 6) apresentação da revisão integrativa. A questão que norteou a pesquisa foi quais as evidências produzidas por estudos que utilizaram a análise espacial como ferramenta de identificação das áreas prioritárias de intervenção para sífilis?

Para seleção dos artigos foi realizada, em fevereiro e março de 2017, uma busca na literatura científica sem limite de tempo nas seguintes bases de dados do sítio da Biblioteca Virtual em Saúde (BVS): Literatura Internacional em Ciências da Saúde/National Library of Medicine (Medline/PubMed), Database of peer-reviewed literature (Scopus), Web of science, Literatura Latino-Americana e do Caribe em Ciências da Saúde (LILACS), Indice Bibliografico Español de Ciencias de la Salud (IBECS), Portal da Biblioteca Cochrane, Biblioteca Virtual Scientific Eletronic Library Online (SciELO), Base de Dados de Enfermería em Español (CUIDEN) e Base de Dados em Enfermagem (BDENF).

A busca nas bases de dados ocorreu mediante o cruzamento dos seguintes descritores em saúde (DeCS): Sífilis, Análise espacial, Sistema de informação geográfica, Educação em saúde e Mapeamento geográfico e seus correspondentes no $\mathrm{Me}$ dical SubjectHeadings (MeSH): Syphilis, Spatial analysis, Geographic information systems, Health education e Geographic mapping (Figura 1).

Como critérios de inclusão foram considerados artigos disponíveis na íntegra publicados em inglês, português ou espanhol e que responderam a pergunta norteadora da pesquisa. Foram 


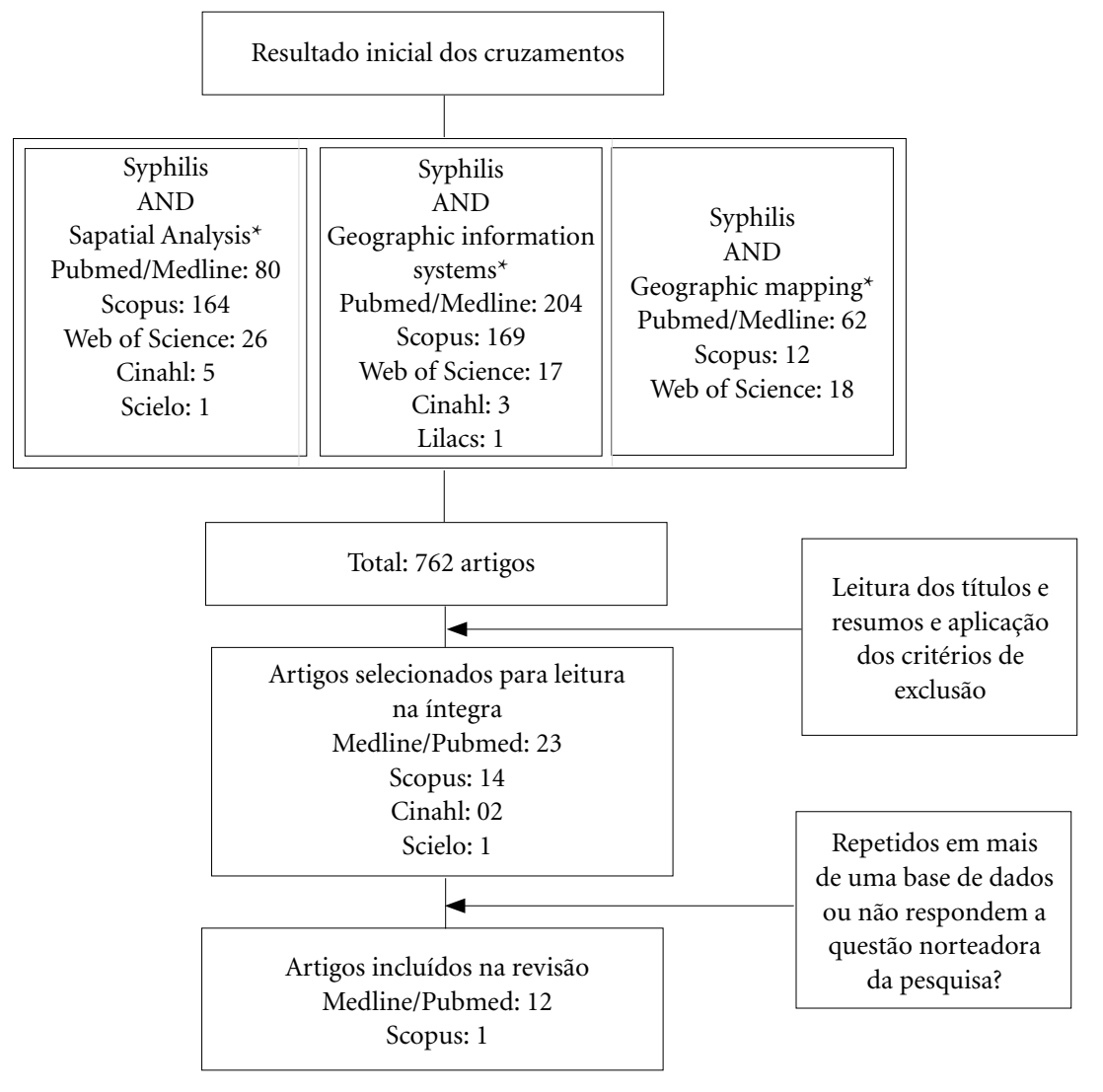

Figura 1. Estratégia de busca utilizada nas bases de dados. Recife, 2017.

${ }^{*}$ As bases e/ou bibliotecas virtuais que não captaram artigos não foram citados.

Fonte: Elaborado pelas autoras.

excluídos artigos repetidos em mais de uma base, resumos de congressos, anais, editoriais, comentários e opiniões, artigos de revisão, teses e dissertações. Para os artigos repetidos, utilizou-se como critério de seleção, o posicionamento da base em relação à seguinte ordem hierárquica: PubMed/Medline, Scopus, Web of Science, Lilacs, Ibecs, Cochrane, SciELO, Cuiden e Bdenf.

Ao final dos cruzamentos "Syphilis (AND) Spatial analysis", "Syphilis (AND) Geographic information systems" e "Syphilis (AND) Geographic mapping", foram encontrados 762 artigos (Figura 1). Após a leitura de todos os títulos e, quando necessário, dos resumos foram pré-selecionados 38 artigos para leitura na íntegra. Desses, foram excluídos 25 pelos seguintes motivos: 14 encontravam-se repetidos em mais de uma base de dados, 2 não estavam disponíveis o texto completo e 9 não responderam a questão norteadora do estudo. Dessa forma, restaram 13 artigos, os quais foram avaliados quanto ao seu rigor metodológico através da aplicação de um instrumento adaptado do Critical Appraisal Skills Programme (CASP) ${ }^{10}$.

O instrumento é composto por 10 itens pontuáveis (máximo de 10 pontos), abrangendo: 1) objetivo do estudo, 2) adequação do desenho metodológico à questão do estudo, 3) justificativa dos procedimentos metodológicos, 4) critérios de seleção da amostra, 5) detalhamento da coleta de dados, 6) relação entre pesquisador e pesquisados, 7) consideração sobre aspectos éticos, 8) rigor na análise dos dados, 9) propriedade na apresentação e discussão dos resultados e 10) valor da pesquisa: apontamento de contribuições, limitações e necessidade de novas pesquisas.

De acordo com esse instrumento os estudos podem ser classificados em duas categorias con- 
forme as seguintes pontuações: 06 a 10 pontos (boa qualidade metodológica e viés reduzido) e mínima de 5 pontos (qualidade metodológica satisfatória, porém com risco de viés aumentado). Nesta revisão, optou-se por utilizar apenas os artigos classificados de 6 a 10 pontos totalizando uma amostra final de 13 artigos, sendo 12 captados nas bases PubMed/Medline e 1 na base Scopus.

O nível das evidências dos artigos foi avaliado considerando sete níveis: o nível 1 representa as evidências provenientes de revisão sistemática ou metanálise de todos os relevantes ensaios clínicos randomizados controlados ou oriundas de diretrizes clínicas baseadas em revisões sistemáticas de ensaios clínicos randomizados controlados; o nível 2 as evidências derivadas de pelo menos um ensaio clínico randomizado controlado bem delineado; o nível 3 as evidências obtidas de ensaios clínicos bem delineados sem randomização; o nível 4 as evidências provenientes de estudos de coorte e de caso-controle bem delineados; o nível 5 as evidências originárias de revisões sistemáticas de estudos descritivos e qualitativos; o nível 6 as evidências derivadas de um único estudo descritivo ou qualitativo e o nível 7 as evidências oriundas de opinião de autoridades e/ou relatórios de comitês de especialistas ${ }^{8}$. A caracterização dos artigos incluídos na revisão está descrita no Quadro 1.

\section{Resultados}

Esta revisão integrativa foi composta por treze artigos, sendo que doze foram captados nas bases Medline/PubMed ${ }^{11-22}$ e apenas um na base Scopus $^{23}$. Todos foram publicados em língua inglesa no período entre 2004 e 2016 nos países China $^{15-17,19,21}$, Estados Unidos ${ }^{11-13,20,23}$, Austrália ${ }^{14}$, África do Sul ${ }^{18}$ e Inglaterra ${ }^{22}$. Após aplicação dos critérios do CASP, todos os artigos foram classificados com boa qualidade metodológica e viés reduzido. Em relação ao nível de evidência, a totalidade dos artigos apresentou nível seis por se tratarem de estudos essencialmente descritivos de cunho epidemiológico.

Em relação à fonte de obtenção dos dados submetidos à análise espacial, a maioria dos artigos utilizou dados secundários ${ }^{11-19,21,23}$, um dos artigos utilizou dados primários (questionário) ${ }^{22}$ e um artigo não citou a fonte ${ }^{20}$. Dentre os artigos caracterizados como ecológicos, dois deles utilizaram o setor censitário como unidade de análise ${ }^{11,23}$, um utilizou condados ${ }^{16}$, um utilizou distritos $^{18}$ e um realizou o estudo utilizando os bairros ${ }^{19}$. As evidências produzidas pelos artigos que compuseram esta revisão estão apresentadas no Quadro 2.

\section{Discussão}

Dos cinco países que publicaram artigos que abordaram a análise espacial da sífilis, a China e os Estados Unidos se destacaram com cinco publicações cada um. No caso da China, esta supremacia de publicações pode ser explicada pela epidemia de sífilis em expansão no país ${ }^{24}$, servindo de incentivo para elaboração de programas abrangentes voltados ao controle e prevenção da doença. Nos Estados Unidos, há também uma crescente notificação de casos de sífilis. Segundo o Departamento de Saúde Norte-Americano, houve um aumento de $10 \%$ do número de contaminações por sífilis entre 2012 e 2013 sendo, $75 \%$ deste aumento decorrente de casos de homens gays e bissexuais. Ao contrário, os estudos de Tan et al. ${ }^{16}$ e Petersen et al. ${ }^{22}$, realizados na China e Inglaterra, respectivamente, encontraram aumento do risco para infecção entre os homens heterossexuais.

$\mathrm{Na}$ maioria dos artigos que compuseram esta revisão ${ }^{11,14,15,17-19,21-23}$ o padrão de distribuição da sífilis ocorreu de forma heterogênea permitindo inferir que planejamentos inespecíficos e uniformes podem não atender a população mais exposta ao risco de acometimento pela doença, bem como onerar custos, na medida em que os esforços estão sendo direcionados para áreas não prioritárias.

A visualização do padrão de distribuição de um evento de saúde através de um mapa bem como a análise de sua relação com outros fatores objetivos e mensuráveis contribui para o estudo da transmissão, disseminação e das ações de controle das doenças e dos agravos de saúde de uma determinada área de abrangência ${ }^{25}$. Além disso, pode fornecer informações para uma avaliação dos estratos sob risco em uma determinada população e identificar os estratos agregados em grupos sociais e áreas geográficas bem definidas permitindo o estabelecimento de ações de prevenção e controle para áreas específicas ${ }^{26}$.

Estudos sobre determinantes sociais de saúde, onde a heterogeneidade é grande, compreender se a variabilidade que ocorre na análise dos dados é por flutuações aleatórias ou por efeito espacial propriamente dito é de extrema relevância. Para isso, o modelo de abordagem baye- 
Quadro 1. Caracterização dos artigos incluídos na revisão. Recife-PE, 2018.

\begin{tabular}{|c|c|c|}
\hline $\begin{array}{c}1^{\circ} \text { autor/Base } \\
\text { de Dados }\end{array}$ & Objetivo & Características metodológicas \\
\hline $\begin{array}{l}\text { Law } \mathrm{DC}^{11} \\
\text { Pubmed/ } \\
\text { Medline }\end{array}$ & $\begin{array}{l}\text { Analisar e mapear a distribuição de quatro doenças } \\
\text { sexualmente transmissíveis notificáveis (clamídea/ } \\
\text { uretrite não gonocócica, gonorréia, sífilis e infecção } \\
\text { pelo HIV, para o Condado de Wake, Carolina do Norte, } \\
\text { para intervenção }\end{array}$ & $\begin{array}{l}\text { Amostra: } 4.553 \text { casos de DST sendo } \\
3 \%(121) \text { casos de sífilis } \\
\text { Estudo Ecológico } \\
\text { Rigor metodológico: } 9\end{array}$ \\
\hline $\begin{array}{l}\text { Gesink Law }{ }^{12} \\
\text { Pubmed/ } \\
\text { Medline }\end{array}$ & $\begin{array}{l}\text { Descrever as mudanças na distribuição espacial da } \\
\text { sífilis antes, durante e após um surto em Baltimore, } \\
\text { MD, usando a a entropia máxima bayesiana (BME), } \\
\text { uma técnica geoestatística moderna } \\
\text { para análise e mapeamento espacial }\end{array}$ & $\begin{array}{l}\text { Amostra: } 6.611 \text { casos de sífilis } \\
\text { primária, secundária e latente } \\
\text { Estudo Descritivo } \\
\text { Rigor metodológico: } 8\end{array}$ \\
\hline $\begin{array}{l}\text { Niccolai } \mathrm{LM}^{13} \\
\text { Pubmed/ } \\
\text { Medline }\end{array}$ & $\begin{array}{l}\text { Descrever a epidemiologia da sífilis precoce entre } \\
\text { os homens de Connecticut, região de prevalência } \\
\text { moderada, em } 2004\end{array}$ & $\begin{array}{l}\text { Amostra: } 55 \text { homens com sífilis } \\
\text { precoce Estudo Descritivo } \\
\text { Rigor metodológico: } 8\end{array}$ \\
\hline $\begin{array}{l}\text { Schleihauf } \mathrm{E}^{14} \\
\text { Pubmed/ } \\
\text { Medline }\end{array}$ & $\begin{array}{l}\text { Elucidar a epidemiologia espacial dos casos de } \\
\text { gonorréia, clamídia e sífilis em Nova Gales do Sul } \\
\text { (NSW) }\end{array}$ & $\begin{array}{l}\text { Amostra: } 7.364 \text { casos de sífilis } \\
\text { Estudo Descritivo } \\
\text { Rigor metodológico: } 8\end{array}$ \\
\hline $\begin{array}{l}\text { Yang } \mathrm{LG}^{15} \\
\text { Pubmed/ } \\
\text { Medline }\end{array}$ & $\begin{array}{l}\text { Descrever as características sociais, demográficas e } \\
\text { geográficas dos casos de sífilis primária notificados, } \\
\text { a fim de segmentar espacialmente os programas de } \\
\text { controle da sífilis e analisar as mudanças espaciais } \\
\text { no desenvolvimento econômico como um único } \\
\text { determinante potencial da variação espacial da sífilis }\end{array}$ & $\begin{array}{l}\text { Amostra: } 52.036 \text { casos de sífilis } \\
\text { primária } \\
\text { Estudo Descritivo } \\
\text { Rigor metodológico: } 9\end{array}$ \\
\hline $\begin{array}{l}\text { Tan } \mathrm{NX}^{16} \\
\text { Pubmed/ } \\
\text { Medline }\end{array}$ & $\begin{array}{l}\text { Examinar a associação entre as características } \\
\text { sociodemográficas e a sífilis e gonorréia na província de } \\
\text { Guangdong }\end{array}$ & $\begin{array}{l}\text { Amostra: } 97 \text { Condados } \\
\text { Estudo Ecológico } \\
\text { Rigor metodológico: } 9 \\
\end{array}$ \\
\hline $\begin{array}{l}\text { Cheng } \mathrm{YJ}^{17} \\
\text { Pubmed/ } \\
\text { Medline }\end{array}$ & $\begin{array}{l}\text { Determinar a distribuição espacial e os fatores de risco } \\
\text { para sífilis na província de Jiangsu, República Popular } \\
\text { da China, durante } 2005 \text { e } 2009 \text { para fornecer estratégias } \\
\text { de prevenção e controle futuro da doença }\end{array}$ & $\begin{array}{l}\text { Amostra: Taxas de incidência de sífilis } \\
\text { primária, secundária e terciária de } \\
2005 \text { a } 2009 \\
\text { Estudo Descritivo } \\
\text { Rigor metodológico: } 8\end{array}$ \\
\hline $\begin{array}{l}\text { Manda SO }{ }^{18} \\
\text { Pubmed/ } \\
\text { Medline }\end{array}$ & $\begin{array}{l}\text { Investigar a associação ecológica entre o vírus da } \\
\text { imunodeficiência humana (HIV) e sífilis na África do } \\
\text { Sul utilizando modelos de mapeamentos conjuntos } \\
\text { para múltiplas doenças }\end{array}$ & $\begin{array}{l}\text { Amostra: } 33.684 \text { mulheres grávidas } \\
\text { (2007), 33.927 (2008) e } 33.861 \text { (2009) } \\
\text { Estudo Ecológico } \\
\text { Rigor metodológico: } 9\end{array}$ \\
\hline $\begin{array}{l}\text { Wu X } \\
\text { Pubmed/ } \\
\text { Medline }\end{array}$ & $\begin{array}{l}\text { Análise multinível e espacial da sífilis em Shenzhen, } \\
\text { na China, para informar medidas de controle } \\
\text { espacialmente direcionadas }\end{array}$ & $\begin{array}{l}\text { Amostra: } 6.496 \text { casos de sífilis } \\
\text { Estudo Ecológico } \\
\text { Rigor metológico: } 9\end{array}$ \\
\hline $\begin{array}{l}\text { Goswami } \\
\text { ND }^{20} \\
\text { Pubmed/ } \\
\text { Medline }\end{array}$ & $\begin{array}{l}\text { Determinar a viabilidade e a taxa de detecção de casos } \\
\text { de uma estratégia integrada de rastreio da tuberculose, } \\
\text { sífilis e vírus da imunodeficiência humana (HIV) } \\
\text { baseada em sistemas de informação geográfica (GIS) }\end{array}$ & $\begin{array}{l}\text { Amostra: } 247 \text { participantes } \\
\text { Estudo Transversal } \\
\text { Rigor metodológico: } 8\end{array}$ \\
\hline $\begin{array}{l}\text { Zhang } \mathrm{W}^{21} \\
\text { Pubmed/ } \\
\text { Medline }\end{array}$ & $\begin{array}{l}\text { Identificar subpopulações demográficas e espaciais que } \\
\text { podem ter uma maior carga de sífilis, para informar } \\
\text { programas de controle mais direcionados }\end{array}$ & $\begin{array}{l}\text { Amostra: } 60.178 \text { casos de sífiliS } \\
\text { Estudo Descritivo } \\
\text { Rigor metodológico: } 8\end{array}$ \\
\hline $\begin{array}{l}\text { Petersen } J^{22} \\
\text { Pubmed/ } \\
\text { Medline }\end{array}$ & $\begin{array}{l}\text { Usar a epidemia de sífilis para desenvolver um método } \\
\text { de identificação e exploração de clusters espaciais e } \\
\text { variações na trajetória de epidemias locais }\end{array}$ & $\begin{array}{l}\text { Amostra: } 12.521 \text { casos de sífilis } \\
\text { Estudo Descritivo } \\
\text { Rigor metodológico: } 7\end{array}$ \\
\hline $\begin{array}{l}\text { Escamilla } \mathrm{V}^{23} \\
\text { Scopus }\end{array}$ & $\begin{array}{l}\text { Avaliar o impacto do método de detecção de cluster } \\
\text { e escala geogrtáfica na identificação das áreas com } \\
\text { alta carga de sífilis no Condado de Mecklenburg, um } \\
\text { Condado em North Carolina }\end{array}$ & $\begin{array}{l}\text { Amostra: } 572 \text { casos geocodificados } \\
\text { de sífilis } \\
\text { Estudo Ecológico } \\
\text { Rigor metodológico: } 8\end{array}$ \\
\hline
\end{tabular}


Quadro 2. Evidências produzidas pelos artigos que utilizaram a análise espacial como ferramenta para identificação das áreas prioritárias de intervenções preventivas para sífilis. Recife-PE, 2018.

\begin{tabular}{|c|c|}
\hline $\begin{array}{l}\text { Número } \\
\text { do artigo }\end{array}$ & Evidências \\
\hline 11 & $\begin{array}{l}\text { Produção de uma imagem mais abrangente do padrão da doença do que a produzida por } \\
\text { entrevistas com profissionais de saúde; Redução de custos na medida em que direcionam as áreas } \\
\text { prioritárias de atuação das equipes de intervenção e podem identificar os surtos emergentes. }\end{array}$ \\
\hline 12 & $\begin{array}{l}\text { A técnica geoestatística de entropia máxima bayesiana (BME) se mostrou útil para descrever } \\
\text { quantitativa e qualitativamente o desenvolvimento espacial e a disseminação da sífilis. }\end{array}$ \\
\hline 13 & $\begin{array}{l}\text { A análise espacial demonstrou que o local de estudo não era prioritário para ações de intervenção } \\
\text { para sífilis, pois não era uma área geoespacial de transmissão da doença. }\end{array}$ \\
\hline 14 & $\begin{array}{l}\text { A área postal de residência é um importante preditor da incidência das doenças sexualmente } \\
\text { transmissíveis. }\end{array}$ \\
\hline 15 & $\begin{array}{l}\text { O local do estudo apresentou-se endêmico para sífilis com áreas de cluster da doença. A aplicação } \\
\text { do índice de Moran para avaliar autocorrelação espacial entre os casos de sífilis e as características } \\
\text { econômicas serviu para demonstrar que os casos estavam agrupados em municípios com maior PIB } \\
(p=0,004) \text { e em alta proporção na população com educação limitada. }\end{array}$ \\
\hline 16 & $\begin{array}{l}\text { As taxas de incidência de sífilis em nível do condado foram significativamente associadas com os } \\
\text { percentuais de homens empregados, divorciados e com padrão de vida mais elevado. A análise } \\
\text { espacial constatou maior incidência de casos nos municípios com padrão mais elevado de vida e } \\
\text { entre os homens heterossexuais. }\end{array}$ \\
\hline 17 & $\begin{array}{l}\text { A análise espacial demonstrou a existência de áreas de clusters da incidência de sífilis no local do } \\
\text { estudo. Demonstrou-se leve associação da incidência de sífilis com as áreas de maior temperatura; } \\
\text { correlação positiva com o transporte demonstrando que os trabalhadores migrantes representavam } \\
\text { um grupo de risco para transmissão e ausência de correlação com o índice de vegetação por } \\
\text { diferença normalizada durante as quatro estações do ano. Foi observado que os recursos hídricos } \\
\text { não podem influenciar a transmissão da sífilis diretamente. }\end{array}$ \\
\hline 18 & $\begin{array}{l}\text { Foi demonstrada correlação negativa entre a sífilis e o HIV, os efeitos da densidade populacional } \\
\text { e do índice de privação (material e social) foram positivamente associados ao HIV, mas } \\
\text { negativamente a sífilis. A análise espacial demonstrou maior prevalência de sífilis nas partes } \\
\text { ocidentais e entre as regiões mais rurais do noroeste do local do estudo. }\end{array}$ \\
\hline 19 & $\begin{array}{l}\text { O estudo demonstrou agrupamento espacial significativo da sífilis primária/secundária e sífilis } \\
\text { latente. No nível individual, a média de idade dos casos de sífilis foi de } 34,3 \text { e as ocupações mais } \\
\text { comuns associadas aos casos da doença foram trabalhadores, desempregados ou dona-de-casa } \\
\text { e serviços empresariais. Ao nível dos bairros, houve considerável heterogeneidade dos casos da } \\
\text { doença com maior propensão a ter sífilis primária/secundária entre os jovens migrantes. Não } \\
\text { houve associação entre os casos da doença e os locais onde se realizam a prática de sexo comercial } \\
\text { e os distritos com maior Produto Interno Bruto estiveram fortemente associdos aos locais com } \\
\text { prevalência mais alta de sífilis. }\end{array}$ \\
\hline 20 & $\begin{array}{l}\text { A análise espacial identificou duas áreas de "hot spots" (áreas com mais e dez casos das três doenças } \\
\text { por período de três anos) no condado estudado. Não houve correlação entre as áreas de maior } \\
\text { densidade populacional com as áreas identificadas como "hot spots". Foi observado que a detecção } \\
\text { integrada de TB, HIV e sífilis baseada em dados geoespaciais é viável. podendo ser um recurso entre } \\
\text { populações com maior carência de atendimento de saúde. }\end{array}$ \\
\hline
\end{tabular}

siana representa uma alternativa aos modelos tradicionais para o estudo de análise espacial de doenças. Nesta revisão, dois dos artigos ${ }^{12,16}$ utilizaram a entropia máxima Bayesiana (BME) e a técnica de alisamento bayesiano, respectivamente, para descrever quantitativa e qualitativamente o desenvolvimento espacial e a disseminação da sífilis. Nessas abordagens, foi possível a geração de estimativas mais precisas de associações entre a incidência da doença e fatores relacionados à área sob investigação.

A análise crítica dos artigos permitiu identificar algumas evidências que podem ser utilizadas para nortear intervenções educativas para prevenção da sífilis ajustadas a cada realidade. Em relação aos aspectos econômicos, quatro artigos apresentaram resultados semelhantes ${ }^{15,16,18,19}$ demonstrando maior agrupamento da doença nas 
Quadro 2. Evidências produzidas pelos artigos que utilizaram a análise espacial como ferramenta para identificação das áreas prioritárias de intervenções preventivas para sífilis. Recife-PE, 2018.

\begin{tabular}{|l|l|}
\hline $\begin{array}{l}\text { Número } \\
\text { do artigo }\end{array}$ & \begin{tabular}{l}
\multicolumn{1}{c|}{ Evidências } \\
\hline 21
\end{tabular} \\
$\begin{array}{l}\text { A análise espacial permitiu a identificação da região epidemiológica com maior fardo da doença e } \\
\text { prioritária para práticas intervencionistas e de controle. Houve menor incidência de casos de sífilis } \\
\text { primária, secundária e latente nos meses de inverno e de sífilis primária e secundária na populaçao } \\
\text { entre 20-39 anos. A maior incidência de sífilis latente foi observada no grupo etário acima de } 20 \\
\text { anos e de sífilis primária e secundária no grupo acima de } 50 \text { anos. Quanto ao estado conjugal, } \\
\text { educação e forma de aquisição mais provável da sífilis, a população casada e com ensino médio } \\
\text { obteve a maior proporção da infecção e aproximadamente a metade dos casos de sífilis latente } \\
\text { foram originalmente infectados através de relações heterossexuais. }\end{array}$ \\
\hline 22 & $\begin{array}{l}\text { A anáise espacial demonstrou que Londres carrega o maior peso dos diagnósticos de sífilis em } \\
\text { termos de incidência, densidade e números absolutos, obtendo uma taxa de incidência cinco } \\
\text { vezes maior do que o resto da Inglaterra no período do estudo. A maioria dos diagnósticos } \\
\text { foram observados em áreas endêmicas; Os clusters espaço-tempo, que incluíam 10\% de todos os } \\
\text { diagnósticos, foram encontrados em áreas urbanas e rurais estando significativamente associados } \\
\text { a uma maior proporção de diagnósticos feitos em homens nascidos no Reino Unido e com idade } \\
\text { inferior a 25 anos e proporções mais baixas de homosexuais, homens co-infectados por HIV e com } \\
\text { idade entre 35-44 anos. }\end{array}$ \\
\hline 23 & $\begin{array}{l}\text { O estudo mostrou maiores taxas de incidência de sífilis primária e secundária no centro de } \\
\text { Charlotte e diminuição das taxas a medida que a distância do Centro aumentava. Foi demonstrado } \\
\text { que a detecção dos clusters de sífilis realizados pela estatística local de Moran e pela estatística de } \\
\text { varredura espacial de Kulldorff podem diferir. }\end{array}$ \\
\hline
\end{tabular}

Fonte: Elaborado pelas autoras.

áreas de maior desenvolvimento econômico. Sobre este achado, um deles ${ }^{15}$ ressaltou como proposta a realização de testes de sífilis nos locais de trabalho nas áreas de maior desenvolvimento econômico. Outro ${ }^{16}$, referiu que esta associação positiva entre os casos de sífilis e o maior nível econômico ocorre devido ao aumento da exposição da população de maior poder aquisitivo a prática do sexo comercial e inseguro, demonstrando que as localidades onde este tipo de prática acontece representa uma zona importante de intervenção.

Um outro artigo ${ }^{18}$ encontrou relação inversa entre o índice de privação social (privação material e social) e a incidência de sífilis, demonstrando que os grupos populacionais de nível econômico menos favorecido está mais exposto a taxas mais elevadas da infecção. E por fim, outro estudo $^{19}$, embora tenha econtrado maior concentração de casos da doença nas áreas de maior Produto Interno Bruto (PIB), não encontrou associação entre os casos da doença e os locais onde se realizam a prática de sexo comercial.

Quanto à educação, três artigos ${ }^{15,16,21}$ encontraram maior proporção de sífilis nas áreas com menores taxas de alfabetização sublinhando a necessidade de educação e intervenções sexuais em sífilis para população de baixa escolaridade.
Interessante ressaltar que apesar ter encontrado maiores taxas de sífilis na população com padrão de vida mais elevado, foi demonstrado forte correlação negativa entre os casos da doença e as taxas de analfabetismo ${ }^{16}$.

Em relação aos aspectos demográficos, um dos $\operatorname{artigos}^{18}$ encontriu relação inversa entre a densidade populacional e a incidência de sífilis enquanto que outro artigo ${ }^{20}$ não encontrou associação entre essas duas variáveis. Quanto a faixa etária, houve variação nas faixas de idade onde foram encontradas maior proporção de sífilis. Em um dos artigos ${ }^{22}$ houve maior proporção da doença entre os homens com idade inferior a 25 anos ou entre 35 e 44 anos. Já em outro artigo ${ }^{21}$ a infecção foi mais prevalente na população com idade entre 20 e 39 anos e acima de 50 anos. Sobre as faixas etárias de maior proporção da sífilis, os estudos demonstraram que a infecção se apresentou mais agrupada tanto na população mais jovem $^{22}$ como na população mais envelhecida ${ }^{21} \mathrm{o}$ que permite inferir que intervenções educativas elaboradas para faixas etárias não prioritárias naquela área determinada, possivelmente não surtirão efeito para o controle da infecção.

Quanto ao estado conjugal, um dos artigos encontrou maior incidência de sífilis na população casada ${ }^{21}$. Outros dois estudos ${ }^{16,22}$ apesar de 
não terem trabalhado a variável estado conjugal, demonstraram que as relações heterossexuais representaram a principal forma de transmissão da doença, resultado que contraria outras literaturas científicas que apontam maior risco para transmissão da infecção nas relações homossexuais $^{27-29}$.

Um dos artigos ressaltou maior agrupamento de sífilis dentre os seguimentos da população de trabalhadores, desempregados ou donas de casa e dentre os que desempenham atividades de cunho empresarial ${ }^{19}$ demonstrando grande diferença entre os grupos que apresentaram maior fardo da doença. Considerando essa diferença, é possível inferir uma dificuldade de elaboração de intervenções educativas capazes de atingir uma população caracteristicamente diversa. Por este motivo, o artigo apresentou como proposta a elaboração de atividades interventivas para promoção da saúde sexual construídas em parceria entre os setores saúde e economia, já que é possível uma relação direta entre o desenvolvimento econômico e a parcela da população que exerce atividades empresariais, citada como grupo de risco.

Outro artigo $^{18}$ demonstrou efeitos ecológicos e espaciais discordantes entre sífilis e HIV sugerindo que a prevalência de uma infecção não pode ser usada como fator preditor da prevalência da outra. Este foi um achado importante por demonstrar que apesar de tratar-se de duas infecções sexualmente transmissíveis, os fatores de risco para ambas as infecções se mostraram distribuídos espacialmente de forma diferente. Portanto, atividades de educação em saúde que generalizem tais fatores, possivelmente não alcançarão o sucesso esperado.

$\mathrm{O}$ indicador composto Índice de Vegetação por Diferença Normalizada, apesar de incomum de ser trabalhado neste tipo de investigação, foi testado para verificação de associação com os locais de agrupamento dos casos de sífilis ${ }^{17}$. Não foi encontrado relação direta deste indicador com a infecção, porém, corroborando como outro artigo $^{21}$ as áreas de maior proporção de casos da doença se apresentaram mais relacionadas as áreas de maior temperatura.

Um aspecto interessante ressaltado por um dos $\operatorname{artigos}^{20}$ foi a viabilidade de uma abordagem geoespacial de rastreio integrado para sífilis, tuberculose e HIV, principalmente para populações com maior carência de atendimento de saúde. Este estudo demosntrou que esta detecção integrada, baseada em dados geoespaciais tem um potencial de detecção significativo para aumentar o impacto de programas organizados por departamentos de saúde com recursos limitados.

Dificuldade de realização de intervenções preventivas direcionadas ou baseadas em locais foi demonstrada por um dos artigos ${ }^{13}$ devido ao estudo ter sido realizado em uma área geoespacial não considerada como de transmissão da sífilis em virtude da dispersão encontrada entre os casos e pela alta proporção de parceiros sexuais que residiam fora do estado estudado. De forma semelhante, outro estudo ${ }^{17}$ encontrou correlação positiva da incidência de sífilis com o transporte demonstrando que os trabalhadores migrantes representavam um grupo de risco para transmissão bem como outra publicação que também ressaltou agrupamento de sífilis entre jovens migrantes ${ }^{19}$. Esses resultados demonstraram que muitos dos parceiros(as) sexuais responsáveis pela transmissão da infecção nos locais onde tais estudos foram realizados eram importados de outros locais, evidência importante proporcionada pela análise espacial a ser considerada na formulação de estratégias de intervenção.

Apesar das evidências encontradas de que a análise espacial pode nortear a construção de estratégias de intervenção em saúde para a prevenção e controle da sífilis, um dos estudos ${ }^{23}$ alertou para importância de se escolher a escala correta para análise e identificação precisa das áreas com alta carga de doença e importância dos analistas e tomadores de decisão estarem atentos ao método e à escala na qual eles identificam clusters, pois isso afetará sua interpretação. Concluiu ainda que uma compreensão mais completa da localização de cluster de alta carga pode informar a alocação de recursos para intervenções de infecção sexualmente direcionada geograficamente.

\section{Conclusão}

Esta revisão permitiu evidenciar que os casos de sífilis se distribuem espacialmente de forma bastante heterogênea, não obedecendo a um perfil epidemiológico único entre os municípios, distritos, cidades, bairros e setores censitários. As evidências apresentadas demonstraram que a análise espacial dos casos da doença representa uma ferramenta eficaz e capaz de direcionar a construção de intervencões educativas específicas para cada realidade evitando, dessa forma, o investimento em áreas geograficamente não prioritparias para o controle da infecção. 


\section{Colaboradores}

RSPS Ramos: planejamento da pesquisa, coleta dos dados, análise e discussão dos resultados, redação do artigo. VP Ramos: revisão crítica e adequação as normas da revista; redação do artigo.

\section{Referências}

1. Brasil. Ministério da Saúde (MS). Secretaria de Vigilância em Saúde. Programa Nacional de DST e Aids. Manual de Controle das Doenças Sexualmente Transmissíveis. Brasília: MS; 2005. [Série Manuais no 68, $4^{\mathrm{a}}$ ed.].

2. Brasil. Ministério da Saúde (MS). Secretaria de Vigilância em Saúde. Protocolo para a prevenção de transmissão vertical de HIV e sifilis - manual de bolso. Brasília: MS; 2007.

3. World Health Organization (WHO). Progress report 2010 [Internet]. [acessado 2017 maio 20]. Disponível em: http://www.who.int./.

4. Brasil. Ministério da Saúde (MS). Secretaria de Vigilância em Saúde. Departamento de DST, AIDS e Hepatites Virais. Boletim Epidemiológico Sifilis. Brasília: MS; 2012.

5. Cavalcante MPR, Oliveira C, Simão FB, Lima PR, Monteiro PS. Análise geoespacial: um estudo sobre a dengue. Acta Paul Enferm 2013; 26(4):360-368.

6. Ferreira RA, Ferriani MGC, Mello DF, Carvalho IP, Cano MA, Oliveira LA. Análise espacial da vulnerabilidade social da gravidez na adolescência. Cad Saude Publica 2012; 28(2):313-323.

7. Holanda ER, Galvão MTG, Pedrosa NL, Paiva SS, Almeida RLF. Análise espacial da infecção pelo vírus da imunodeficiência humana entre gestantes. Rev Latino -Am Enferm 2015; 23(3):441-449.

8. Melnyk BM, Fineout-Overholt E. Making the case for evidence-based practice. In: Melnyk BM, Fineou$\mathrm{t}$-Overholt E. Evidence-based practice in nursing \& healthcare. A guide to best practice. $2^{\text {a }}$ ed. Philadelphia: Lippincot Williams \& Wilkins; 2011. p. 3-24.

9. Glasziou P, Del Mar C, Salisbury J. Prática Clínica Baseada em Evidências: Livro de Exercícios. 2a ed. Porto Alegre: Artemed; 2010.

10. Milton K. Primary Care Trust. Critical Apprasisal Skills Programme [Internet]. London: Oxford; 2002. [acessado 2017 maio 20]. Disponível em: http://www.columbia.edu/ mvp19/RMC/M3/CASP_Qual.pdf.

11. Law DC, Serre ML, Christakos G, Leone PA, Miller WC. Spatial analysis and mapping of sexually transmitted diseases to optimise intervention and prevention strategies. Sex Transm Infect 2004; 80(4):294-299.

12. Gesink Law DC, Bernstein KT, Serre ML, Schumacher CM, Leone PA, Zenilman JM, Miller WC, Rompalo AM. Modeling a syphilis outbreak through space and time using the Bayesian maximum entropy approach. Ann Epidemiol 2006; 16(11):797-804.

13. Niccolai LM, Stephens N, Jenkins H, Richardson W, Muth SQ, Rothenberg R. Early syphilis among men in Connecticut: epidemiologic and spatial patterns. Sex Transm Dis 2007; 34(3):183-187.

14. Schleihauf E, Watkins RE, Plant AJ. Heterogeneity in the spatial distribution of bacterial sexually transmitted infections. Sex Transm Infect 2009; 85(1):45-49.

15. Yang LG, Tucker JD, Yang B, Shen SY, Sun XF, Chen YF, Chen XS. BMC. Primary syphilis cases in Guangdong Province 1995-2008: opportunities for linking syphilis control and regional development. Public Health 2010; 10:793.

16. Tan NX, Messina JP, Yang LG, Yang B, Emch M, Chen XS, Cohen MS, Tucker JD. A spatial analysis of county-level variation in syphilis and gonorrhea in Guangdong Province, China. PLoS One 2011; 6(5):e19648. 
17. Cheng YJ, Norris J, Bao CJ, Liang Q, Hu JL, Wu Y, Tang FY, Liu WD, Ding KQ, Zhao Y, Peng ZH, Yu RB, Wang $\mathrm{H}$, Shen HB, Chen F. Geographical information systems-based spatial analysis and implications for syphilis interventions in Jiangsu province, People's Republic of China. Geospat Health 2012; 7(1):63-72.

18. Manda SO, Lombard CJ, Mosala T. Divergent spatial patterns in the prevalence of the human immunodeficiency virus (HIV) and syphilis in South African pregnant women. Geospat Health 2012; 6(2):221-231.

19. Wu X, Tucker JD, Hong F, Messina J, Lan L, Hu Y, Feng T, Emch ME, Liu X, Zhang C, Wen L. Multilevel and spatial analysis of syphilis in Shenzhen, China, to inform spatially targeted control measures. Sex Transm Infect 2012; 88(5):325-329.

20. Goswami ND, Hecker EJ, Vickery C, Ahearn MA, Cox GM, Holland DP, Naggie S, Piedrahita C, Mosher A, Torres Y, Norton BL, Suchindran S, Park PH, Turner D, Stout JE. Geographic information system-based screening for TB, HIV, and syphilis (GIS-THIS): a cross-sectional study. PLoS One 2012; 7(10):e46029.

21. Zhang W, Du Z, Tang S, Guo P, Ye X, Hao Y. Syphilis in the economic center of South China: results from a real-time, web-based surveillance program. BMC Infect Dis 2015; 15:318.

22. Petersen J, Gibin M, Sile B, Simms I. Identifying and interpreting spatiotemporal variation in diagnoses of infectious syphilis among men, England: 2009 to 2013. Sex Transm Infect 2016; 92(5):380-386.

23. Escamilla V, Hampton KH, Gesink DC, Serre ML, Emch M, Leone PA, Samoff E, Miller WC. Influence of detection method and study area scale on syphilis cluster identification in North Carolina. Sex Transm Infect 2016; 43(4):216-221.

24. Wong NS, Huang S, Chen L, Zhao P, Tucker JD, Yang LG, Goh BT, Zheng H, Yang B. Spatiotemporal clusters of primary and secondary syphilis cases in south China: an observational study. Lancet 2016; 388(Supl. 1):S90.
25. Nardi SMT, Paschoal JAA, Pedro HSP, Paschoal VDA, Sichieri EP. Geoprocessamento em Saúde Pública: fundamentos e aplicações. Rev Inst Adolfo Lutz 2013; 72(3):185-191.

26. Carvalho MS, Santos RS. Análise de dados espaciais em saúde pública: métodos, problemas, perspectivas. Cad Saude Publica 2005; 21(2):361-378.

27. Gomes NCRC, Meier DAP, Pieri FM, Alves E, Albanese SPR, Lentine EC, Arcêncio RA, Dessunti EM. Prevalence and factors associated with syphilis in a Reference Center. Rev Soc Bras Med Trop 2017; 50(1):27-34.

28. Nokhodian Z, Yazdani MR, Yaran M, Shoaei P, Mirian M, Ataei B, Babak A, Ataie M. Prevalence and risk factors of HIV, Syphilis, Hepatites B and C among female prisioners in Isfahan, Iran. Hepat Mon 2012; 12(7):442-447.

29. Campos ALA, Araújo MAL, Melo SP, Andrade RFV, Gonçalves MLC. Sífilis em parturientes: aspectos relacionados ao parceiro sexual. Rev Bras Ginecol Obstet 2012; 34(9):397-402.

Artigo apresentado em 26/07/2019

Aprovado em 24/11/2019

Versão final apresentada em 27/11/2019

Editores-chefes: Romeu Gomes, Antônio Augusto Moura da Silva 